\title{
Orbitofrontal cortex and dynamic filtering of emotional stimuli
}

\author{
RANDALL R. RULE, ARTHUR P. SHIMAMURA, and ROBERT T. KNIGHT \\ University of California, Berkeley, California \\ and Veterans Affairs Health Care System, Martinez, California
}

\begin{abstract}
Event-related potentials (ERPs) were recorded in response to mildly aversive somatosensory and auditory stimuli. Patients with orbitofrontal lesions exhibited enhanced ERPs (i.e., P3 amplitudes), as compared with control subjects. Moreover, these patients did not habituate to somatosensory stimuli across blocks of trials. The results were specific to orbitofrontal damage, since patients with damage to the dorsolateral prefrontal cortex did not exhibit enhanced P3 amplitudes. These findings suggest that damage to the orbitofrontal cortex impairs the ability to modulate or inhibit neural responses to aversive stimuli. The findings are couched in terms of dynamic filtering theory, which suggests that the orbitofrontal cortex is involved in the selection and active inhibition of neural circuits associated with emotional responses.
\end{abstract}

Since the classic neurological case of Phineas Gage (Harlow, 1848, 1868; Macmillan, 1986, 1999), damage to the orbitofrontal cortex (OFC) has been linked to impairment in the control and regulation of emotion. Behavioral symptoms associated with such impairment include emotional outbursts, impulsivity, aberrant risk-taking behavior, and socially inappropriate behavior (see Damasio, 1998; Rolls, Hornak, Wade, \& McGrath, 1994; Shimamura, 2000 , in press). In modern times, damage to the OFC is a frequent concomitant of traumatic head injury, such as that incurred in a severe automobile accident. The OFC is particularly sensitive to head trauma, since it is adjacent to bony ridges that make up the skull's openings for the eyes. Shearing against these bony ridges produces contusions in both the OFC and the anterior temporal cortex (Mattson \& Levin, 1990).

Neuropsychological studies of patients with OFC lesions suggest a psychological disorder of emotional disinhibition. In both human and monkey studies, OFC lesions cause aberrant or overly sensitive responses to emotionally laden stimuli. Such disinhibitive responses result in inappropriate actions to prepotent stimuli, overt aggressive behavior, and failure to appreciate reward feedback (Butter, McDonald, \& Snyder, 1969; Dias, Robbins, \& Roberts, 1996; Iversen \& Mishkin, 1970; Rolls et al., 1994). In addition, Damasio and colleagues have shown that patients with OFC lesions elicit abnormal risk-taking behavior, which is linked with reduced galvanic skin responses to high-gamble situations (Bechara, Tranel, Damasio, \&

This research was supported by NIH Grants DA14110 to A.P.S. and NS21135 to R.T.K. and by funding from the Veterans Administration Research Service. The authors thank Donatella Scabini and Clay Clayworth for advice and technical support. Address correspondence to A. P. Shimamura, Department of Psychology (MC1650), University of California, Berkeley, CA 94720-1650(e-mail: aps@ socrates.berkeley.edu).
Damasio, 1996; Damasio, Tranel, \& Damasio, 1990). Taken together, these findings are consistent with the view that the OFC is intricately involved in regulating arousal during emotional events.

Analyses of the neural bases of affective behavior have suggested a broad array of structures and circuits associated with an organism's response to emotional events (for reviews, see Davidson, Putnam, \& Larson, 2000; Panksepp, 1998). For example, subcortical structures, such as the autonomic nervous system, the hypothalamus, and the amygdala, contribute to the induction, activation, encoding, and elicitation of emotion. The amygdala has been viewed as a mediator or interface between primary and secondary (i.e., neocortical) responses to emotional events (LeDoux, 2000; McGaugh, 2000). It is proposed that the OFC is central in the top-down regulation of these neural responses.

The OFC is generally defined as cortical tissue on the ventral (i.e., orbital) surface of the frontal lobes. In humans, this region includes parts of Brodmann areas BA11, BA12, and BA47. On the basis of modern cytoarchitectonic analyses, Petrides and Pandya (1994) designated the OFC as parts of areas PP11, PP13, PP14, and PP47/12 (we added the "PP" to distinguish this nomenclature from that of Brodmann). The OFC has also be described as the ventromedial prefrontal cortex (Damasio, 1998). We use the term orbitofrontal cortex, since it succinctly describes the neurologicalimpairment associated with patients assessed in this study. That is, lesions in these patients are situated on the orbital surface, particularly centered on BA11 (i.e., PP14). Moreover, lesions in these patients may also encroach on the ventrolateral prefrontal cortex (BA47 or PP47/12), and thus the term ventromedial prefrontal cortex is not as accurate for these patients as the more general term orbitofrontal cortex.

Various mechanisms have been proposed to account for the role of the OFC in regulating emotion (Damasio, 1998; 
Rolls, 2000; Shimamura, 2000). Damasio (1998) described a "somatic marker" hypothesis, in which the OFC is critical in representing somatosensory (feedback) states associated with emotional events. By this view, patients with OFC lesions exhibit deficits in emotional regulation because they fail to encode or represent bodily sensations (i.e., somatic markers) related to emotionally charged events (Damasio et al., 1990). A related view by Rolls (2000) suggests that the OFC is involved in assessing and representing learned contingencies of reinforcement (i.e., how reward or punishment is associated with an event). In other words, the OFC is critical in the appreciation of the hedonic value (good vs. bad) of one's actions. Failure to appreciate reinforcement contingencies can lead to disinhibitive or socially inappropriate behavior, since emotionally laden behavior may be elicited without regard to possible consequences. Theoretical views by Damasio (1998) and Rolls (2000) suggest that OFC damage causes an impoverished or reduced neural response to an emotional event. During an emotional event, the OFC would normally signal to the organism information about the consequences (pleasant/unpleasant) of one's actions.

We propose dynamic filtering theory as a neural mechanism to account for the role of the OFC in emotional regulation. We suggest that the OFC acts to filter or gate neural activity associated with an arousing event. That is, the OFC - by way of inputs from other cortical and subcortical areas-monitors the plethora of neural responses associated with an emotional event. With this information, the OFC initiates control via reciprocal efferent projections that are used to maintain task-relevant activations and inhibit irrelevant or inappropriate neural activity (Shimamura, 2000). By this account, the OFC does not induce emotions, nor does it fully represent emotional events. Instead, it monitors and controls emotional responses initiated by other brain regions. This neural mechanism is identical to the kind of gating or filtering mechanism associated with the dorsolateral prefrontal cortex (DLPFC) in its capacity to monitor and control cognition (see Knight, Staines, Swick, \& Chao, 1999; Shimamura, 2000). The critical difference is that the OFC controls emotional processes, whereas the DLPFC controls perceptual and memory processes. Thus, the prefrontal cortex as a whole is involved in executive control of information processing in many domains (D'Esposito, Postle, Ballard, \& Lease, 1999; Knight et al., 1999; Miller \& Cohen, 2001; Petrides, 1998; Shallice \& Burgess, 1993; Shimamura, 2000; Smith \& Jonides, 1999). On the basis of dynamic filtering theory, the nature of control (emotion, perception, memory) depends on the specific anatomical connections between prefrontal regions and other brain regions (Shimamura, 2000).

With respect to emotional control, suppression of emotional arousal is often demanded in certain social situations (e.g., interacting with a superior or a mate). Failure to suppress emotional arousal leads to the kind of disinhibitive responses associated with OFC damage, such as impulsivity and socially inappropriate behavior. On the basis of dynamic filtering theory, OFC damage results in a failure to gate neural activity associated with emotional stimuli. Thus, OFC damage should result in increased or overly responsive neural activity in other brain regions associated with emotional responses. This prediction is critical for dynamic filtering theory, but it is not suggested by the somatic marker hypothesis or Roll's learned reinforcement theory.

In the present study, we assessed neural activity in response to emotionally arousing stimuli, such as mildly aversive shocks and abrupt sounds. Scalp-recorded eventrelated potentials (ERPs) were measured during the presentation of these mildly aversive stimuli. We assessed patients with OFC lesions, patients with DLPFC lesions, and age-matched neurologically intact control subjects for each patient group. We were particularly interested in differences among these groups in the amplitude of ERPs to these emotionally laden stimuli. Moreover, we assessed the degree to which such responses habituate over time.

\section{METHOD}

\section{Subjects}

The OFC patient group consisted of 4 male subjects with bilateral lesions involving the orbital prefrontal cortex (Figure 1). Three of these individuals incurred brain injury following closed head trauma. In the 4th case, orbitofrontal lesion was the result of surgery to remove a meningioma. Lesion site was determined by examination of MRI scans. All had extensive bilateral damage centering in BA11 and no damage to either the dorsolateral frontal cortex or the basal forebrain area. The lesions in these patients covered both the ventromedial cortex (i.e., the orbitofrontal gyrus and the medial frontal cortex) and the ventrolateral cortex (i.e., the inferior frontal gyrus). The mean age of this patient group was 44 years. These patients exhibited typical behavioral profiles associated with OFC lesions, including impulsivity and socially inappropriate behavior.

Five patients with DLPFC lesions that spared the ventral prefrontal regions were also tested ( 3 males, 2 females). These patients were assessed primarily to determine whether findings from OFC patients were specific to the OFC or whether they were generalized to other prefrontal regions. These DLPFC patients were representative of patients that have been tested extensively in neuropsychological and electrophysiological studies of working memory (Baldo \& Shimamura, 2000; Chao \& Knight, 1996). All the patients had unilateral damage to the DLPFC resulting from cerebrovascular stroke in the anterior branch of the middle cerebral artery. Lesion site, as determined by MRI scans, was centered on BA46 and BA9 and spared the OFC. The mean age for this group was 75 years.

Since the two patient groups differed considerably in age, two separate age-matched control groups were assessed. The control group for the OFC patients consisted of 5 neurologically intact individuals ( 3 males and 2 females) with a mean age of 45 years (no significant differences in ERP responses were observed between the male and the female subjects used in this study). The control group for the DLPFC patients consisted of 5 neurologically intact individuals ( 3 males and 2 females) with a mean age of 66 years. Control subjects were volunteers from the Veterans Affairs Medical Center in Martinez, California. Informed consent, approved by both the VA and the University of California, Berkeley Institutional Review Board, were obtained from all the subjects.

\section{Design and Apparatus}

A total of four blocks were presented (18 min per block), with each block consisting of 36 somatosensory and 36 auditory stimuli. 


\section{r.v.}
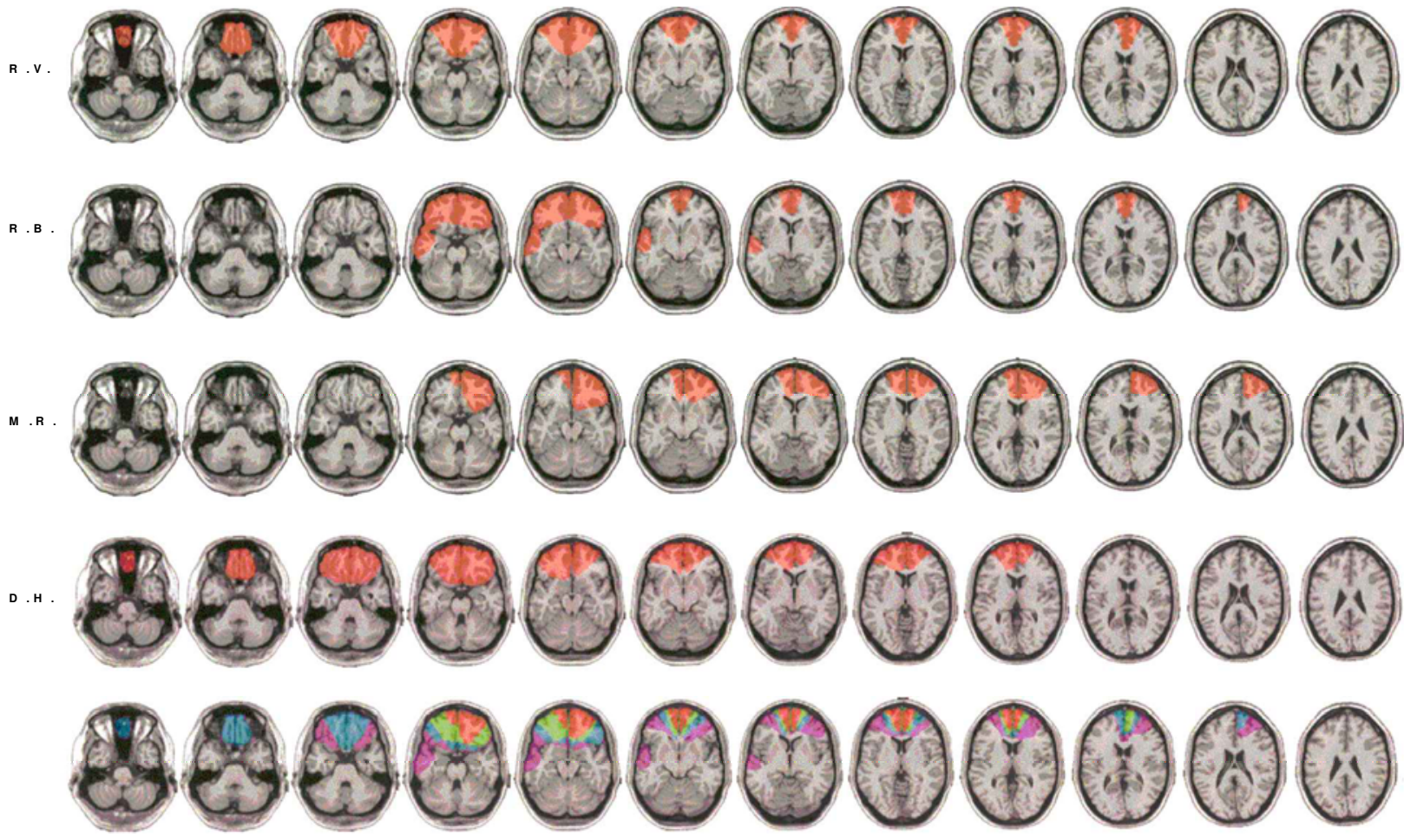

$0 \quad 50 \quad 100 \%$

Figure 1. Extent of lesions in orbitofrontal cortex patients $(n=4)$ as reconstructed from CT scans. Each row shows the extent of damage in an individual patient as transcribed onto axial templates, using 5 -mm cuts. The bottom row represents average extent of overlap across the 4 patients (percentage of overlap is indicated by color code).

The somatosensory stimuli consisted of mild electrical shocks delivered to the wrist by a Grass $\mathrm{S} 88$ constant current stimulator. The shocks were square pulses of $0.2-\mathrm{msec}$ duration, calibrated for each subject to obtain a visible twitch of the abductor pollicus brevis muscle. The average voltage necessary to elicit a twitch did not differ between groups. Half of the somatosensory stimuli were administered to the left wrist, and the other half were administered to the right wrist.

The set of auditory stimuli consisted of 144 environmental sounds (e.g., train whistle, dog bark). Each sound was unique and thus was presented only once during the experiment. The arousing nature of the sounds was assumed to be a function of novelty and abruptness of occurrence. The sounds were $700 \mathrm{msec}$ in duration and were presented with an intensity of 70-75 dB. They were presented monaurally through headphones, with half of the sounds presented to the left ear and the other half presented to the right ear. Order of stimuli (somatosensory vs. auditory) and location of stimuli (left vs. right) were randomized. To maintain a relatively constant level of arousal, the subjects watched a movie with the audio off (Il Postino, with subtitles) during stimulus presentation.

We obtained EEG recordings $(0.01-100 \mathrm{~Hz} ; 1,024$ digitizing rate) with $\mathrm{Ag} / \mathrm{AgCL}$ electrodes placed at three midline scalp locations: $\mathrm{Fz}, \mathrm{Cz}$, and $\mathrm{Pz}$. These electrode sites were referred to a linked mastoid reference. Since the experimental design required two sensory modalities (somatosensory vs. auditory), two fields of presentation (left vs. right), and two patient groups (one with bilateral damage and one with unilateral damage), we selected the most conservative, yet general, array of electrode placement (midline $\mathrm{Fz}, \mathrm{Cz}$, and $\mathrm{Pz}$ ). Prior studies have demonstrated that $\mathrm{P} 3$ activity can be reliably mon- itored from these midline electrodes (Knight, 1984; Knight, Scabini, Woods, \& Clayworth, 1989). Eye movements were monitored with electrooculograms (EOGs) recorded with bipolar electrodes. Vertical EOG was acquired from electrodes placed above and below the right eye, and horizontal EOG was acquired from electrodes placed lateral to each eye. Trials with excessive EOG or electromyogram artifacts $(>100 \mu \mathrm{V})$ were rejected. Peak amplitudes for P3s (240$400 \mathrm{msec}$ ) were measured to a $100-\mathrm{msec}$ prestimulus interval. Latency was recorded relative to stimulus onset. Since comparable results were obtained for left and right stimulation, data analyses were based on mean activity across left and right sites for both auditory and somatosensory stimulation.

\section{Procedure}

The subjects were seated comfortably in a reclining armchair in a dimly lit, electrically and acoustically shielded room. Recording electrodes were then placed on the subject. After calibrating stimulus intensity for somatosensory presentations, the subjects put on headphones and began watching the movie (with the audio turned off). The subjects were instructed simply to sit passively and watch the movie while a block of stimulus trials was presented. After each block, a short 1- to 2-min break was given while the experimenter set up the next block of trials. The entire session lasted approximately $90 \mathrm{~min}$.

\section{Results}

Since primary analyses concerned comparisons between OFC patients and age-matched control subjects, we first 
A u d ito ry

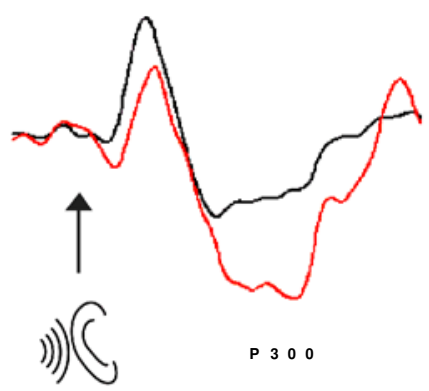

So m a tos e n sory
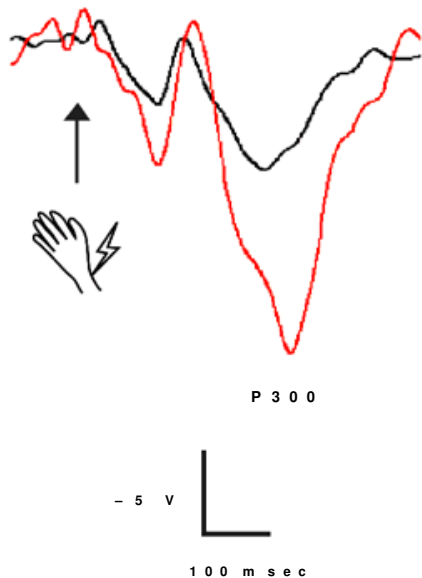

Figure 2. ERP recordings at $\mathrm{Pz}$ site for abrupt auditory (left panel) and somatosensory (right panel) stimuli. Orbitofrontal cortex (OFC) patients (red) exhibited heightened $P 3$ amplitudes, as compared with OFC control subjects (black).

describe results from these two groups. Figure 2 displays averaged ERPs in response to auditory (left panel) and somatosensory (right panel) stimuli. As is shown in these graphs, $\mathrm{P} 3$ amplitudes in response to these stimuli were potentiated in patients with OFC lesions. Specifically, for somatosensory stimuli, the mean P300 amplitude at the Pz electrode was $25.8 \mu \mathrm{V}$ for OFC patients and $11.3 \mu \mathrm{V}$ for control subjects $[F(1,7)=25.94, p<.005]$. The difference between groups was so marked that there was no overlap between the groups: All of the OFC patients exhibited larger P3 amplitudes than did any of the control subjects. For auditory stimuli, the mean P300 amplitude at the Pz electrode was $21.0 \mu \mathrm{V}$ for OFC patients and $9.7 \mu \mathrm{V}$ for control subjects $[F(1,7)=10.54, p<.05]$. We also observed significant group differences at the central $(\mathrm{Cz})$ electrode in response to somatosensory stimuli [OFC patients $=21.2 \mu \mathrm{V} ;$ controls $=13.1 \mu \mathrm{V} ; F(1,7)=17.74$, $p<.005]$.

Habituation of ERP responses was assessed by examining changes in $\mathrm{P} 3$ amplitude across the four blocks of trials. As is shown in Figure 3, control subjects exhibited a significant decrease in $\mathrm{P} 3$ amplitude at the Pz electrode across the four blocks for somatosensory stimuli $[F(3,12)=$ $3.54, p<.05]$. A trend analysis revealed a significant linear effect in amplitude decrease $[F(1,12)=9.78, p<.01]$. Habituation in P3 amplitude was not observed in OFC patients. With respect to auditory stimuli, no significant differences in habituation were observed at the Pz electrode between controls and OFC patients (see Figure 3). Yet, control subjects exhibited a marginally significant habituation effect at the $\mathrm{Cz}$ electrode $[F(3,12)=3.30, p=$
.058]. Further trend analyses revealed a significant linear decrease between blocks $[F(1,4)=9.09, p<.05]$. For auditory stimuli, both groups appeared to exhibit some decrease in signal after the first block, but this effect was not significant.

Comparisons between DLPFC patients and agematched control subjects revealed a very different pattern of response. For somatosensory stimuli, DLPFC patients exhibited a significantly reduced $\mathrm{P} 3$ at the Pz electrode, as compared with control subjects (see Figure 4). Specifically, the mean P3 amplitude of DLPFC subjects was $5.31 \mu \mathrm{V}$, whereas that of the age-matched control group was $9.67 \mu \mathrm{V}$ $[F(1,7)=7.76, p<.05]$. In the present study, ERPs to auditory stimuli were comparable to those exhibited by the control subjects. Patterns of reduced orienting responses in patients with DLPFC lesions have been reported previously (Knight, 1984) and suggest that the disinhibitedERPs observed in OFC patients is not the result of generalized prefrontal damage.

\section{DISCUSSION}

In this study, OFC patients exhibited enhanced $\mathrm{P} 3$ responses to abrupt, emotionally laden stimuli. This effect was observed for both somatosensory and auditory stimuli. As is shown in Figure 4, OFC patients exhibited considerably disinhibited P3 amplitudes, as compared with neurologically intact control subjects and patients with DLPFC lesions. In addition, P3 amplitudes across blocks in patients with OFC lesions did not habituate to the same degree as with control subjects. The failure to habituate 
H a bitu a tion a t P z

som a tosensory control

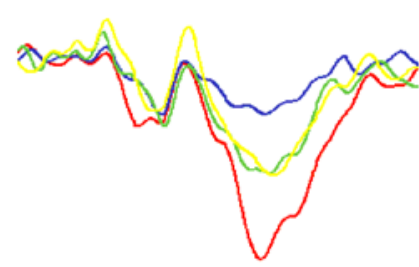

A u d itory
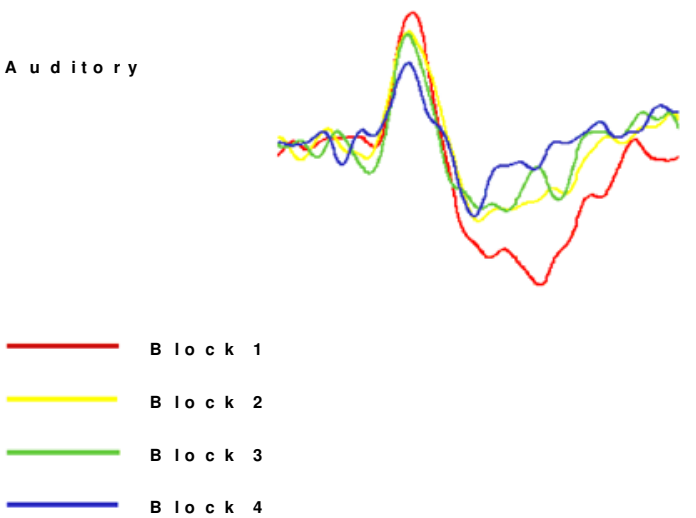

orbitofronta I
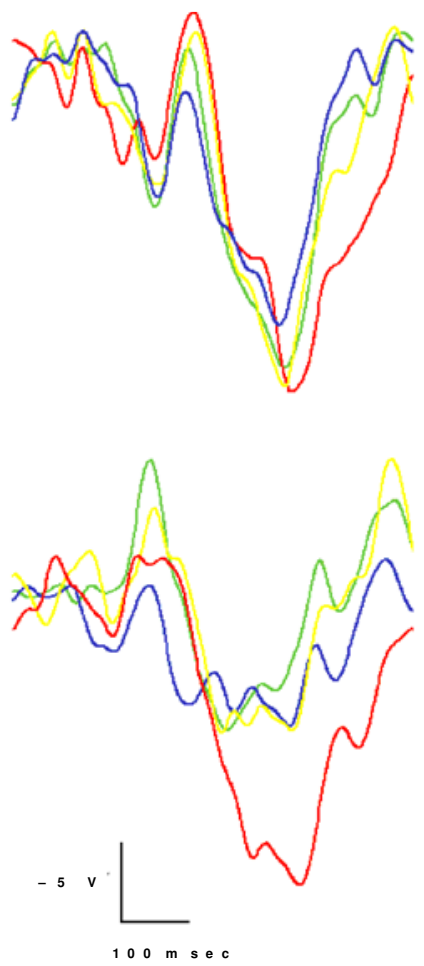

Figure 3. ERP recordings for each block of trials for (A) somatosensory and (B) auditory stimuli. Across the four consecutive blocks of trials, control subjects exhibited reduced ERP responses (i.e., habituation). Orbitofrontal cortex patients failed to habituate to somatosensory (wrist shock) stimuli.

was pronounced for somatosensory stimuli. These findings represent the first electrophysiological evidence of potentiated neural responses to emotionally laden stimuli in patients with well-defined lesions of the OFC.

The results suggest that the OFC plays a role in regulating neural activity associated with emotional stimuli. These findings are consistent with dynamic filtering theory and with previous findings from studies of nonhuman primates. For example, Macaque monkeys with orbitofrontal lesions fail to habituate to novel auditory and visual stimuli (Butter, 1964). Also, performance in go-no-go tasks in both humans and nonhuman primates suggests a particular deficit in inhibiting responses on no-go trials (Dias et al., 1996; Iversen \& Mishkin, 1970; Rolls et al., 1994). These findings are also consistent with behavioral analyses of patients with OFC lesions, such as Phineas Gage and numerous cases since him, who exhibit disinhibition in the expression of emotions, such as being easily angered or making socially inappropriate remarks.

Both the increased P3 amplitude and the lack of habituation were most prominent at the Pz electrode. This posterior scalp P300 response has been shown from lesion studies, intracranial EEG recordings, and fMRI data to be generated in the temporal parietal junction and is part of a multimodal attention network that includes the DLPFC, the cingulate, the temporal-parietal junction, and the posterior hippocampus (Knight, 1984, 1996; Knight et al., 1989; Opitz, Mecklinger, Friederici, \& von Cramon, 1999; for a review, see Soltani \& Knight, in press). For these responses, latency differences were not observed between groups, nor were they observed as a function of habituation within groups. Although effects were maximal at the Pz electrode, significant differences were also observed at the $\mathrm{Cz}$ electrode in response to somatosensory stimuli. Thus, the findings suggest that a consequence of OFC damage is rather pervasive disinhibited activity in posterior cortical regions.

Patients with OFC lesions failed to habituate to somatosensory stimuli. This effect, however, was not observed for auditory stimuli. Two factors may have accounted for the lack of habituation to auditory stimuli. First, the somatosensory stimuli involved wrist shocks, which may have initially been more aversive and emotionally laden than were the abrupt sounds. Second, the same somatosensory stimulus was repeated throughout the test session (i.e., same intensity of wrist shock). The auditory stimulus set 


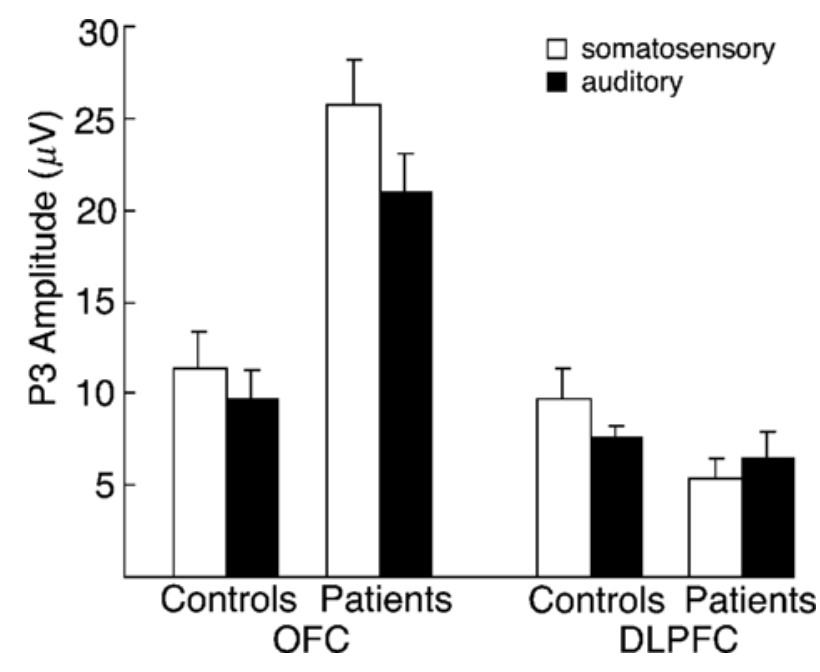

Figure 4. Mean P3 amplitude to somatosensory and auditory stimuli for orbitofrontal cortex (OFC) patients and controls and for dorsolateral prefrontal cortex (DLPFC) patients and controls. As compared with the other groups, the OFC patients exhibited grossly disinhibited $P 3$ responses.

consisted of unique and unpredictable sounds. Thus, the initially intense but repeated somatosensory stimuli may have been more sensitive to habituation than were the unique sounds.

Importantly, these findings suggest regional specificity within the prefrontal cortex. We observed disinhibition of evoked responses to emotional stimuli in the patients with OFC lesions, but not in the patients with the DLPFC lesions. Indeed, the DLPFC patients exhibited normal or even decreased P300, a finding that has been previously reported (Knight, 1984). These findings are not consistent with theories suggesting that OFC lesions produce decreased or impoverished brain responses associated with emotional stimuli. For example, the somatic marker hypothesis focuses on reduced representation of emotional events and reduced neural responses of reactivity (Damasio, 1998). However, it should be noted that reduced $a u$ tonomic, rather than central, responses have been observed in previous studies. One could imagine that reduced peripheral and disinhibited central reactivity together contribute to the disordered behavior observed in these patients.

Our results do agree with the general concept proposed previously by Damasio (1998) and Rolls (2000) that OFC is involved in integrating or associating sensory events to emotional responses. We propose that a failure to modulate (i.e., select and inhibit) neural activity associate with emotional events leads to less refined or less context-bound associations. That is, a filtering deficit leads to greater interference (increased noise) and thus reduces contextual memory for emotional events. This problem is analogous to attentional or working memory problems associated with DLPFC lesions, which lead to problems in inhibiting prepotent memory associations (see Shimamura, 2000). With respect to OFC lesions, the ability to associate sensory events to their hedonic value is affected. This prob- lem could lead to disorders in remembering the affective quality of prior emotional experiences and thus could produce diminished anticipatory responses, such as failure to exhibit heightened heart rate or GSR to stimuli previously associated with emotional consequences (Bechara, Damasio, \& Damasio, 2000; Damasio et al., 1990; Roberts et al., 2001).

Other recent studies have reported findings of increased orienting P300 amplitude responses to both novel (Solbakk, Reinvang, Nielsen, \& Sundet, 1999) and emotional (Kaipio et al., 1999) stimuli following head trauma. These observations are consistent with our findings. Although the lesion analyses in these studies are not fully documented, OFC damage is common in patients with head trauma. Additional findings from our laboratory suggest that these ERP enhancements are not a result of changed conductivity in the damaged brain. For instance, OFC patients have normal posterior $\mathrm{P} 300$ amplitudes in other, nonemotional tasks (Hartikainen, Ogawa, Soltani, Pepitone, \& Knight, 2000).

Although the P300 is thought to be a central component (Knight, 1984) of orienting, enhancement of earlier changes in the ERP waveform was observed in response to the somatosensory stimuli. Both normal subjects and OFC patients generated a negative potential at approximately $150 \mathrm{msec}$ (N150). This negative ERP peak was maximal at frontocentral scalp sites and was generated in the unimodal association cortex (Puce et al., 1995). The OFC patients exhibited larger N150s in response to somatosensory stimuli. This N150 enhancement was significant at the Fz electrode $[F(1,7)=9.31, p=.05]$. In response to auditory stimuli, both groups exhibited a large negativity at $100 \mathrm{msec}$ (N100). OFC damage did not enhance this ERP component. Patients and controls exhibited comparable auditory N100 amplitudes at Fz and Pz. It appears that, at least in the case of somatosensory information, the OFC may exert inhibitory control quite early in the information-processing stream.

Dynamic filtering theory offers a neural account of prefrontal function with respect to monitoring and controlling information processing (see Shimamura, 2000). Although this mechanism explains a prominent feature of prefrontal function, it is important to note that other processes, such as novelty detection and context setting, may be intricately related to the workings of the prefrontal cortex. Nevertheless, the notion of dynamic filtering offers a general mechanism of prefrontal function, and yet it honors dissociations or regional specificity between prefrontal regions. That is, dynamic filtering operates in many prefrontal regions, but each region is controlling different neural circuits and, thus, regulating different aspects of mental function. With respect to emotions, OFC lesions lead to dysregulated or disinhibited neural activity to emotionally laden stimuli.

\section{REFERENCES}

Baldo, J. V., \& Shimamura, A. P. (2000).Spatial and color working memory in patients with lateral prefrontal cortex lesions. Psychobiology, 28, 156-167. 
Bechara, A., Damasio, H., \& Damasio, A. R. (2000). Emotion, decision making and the orbitofrontal cortex. Cerebral Cortex, 10, 295307.

Bechara, A., Tranel, D., Damasio, H., \& Damasio, A. R. (1996). Failure to respond autonomically to anticipated future outcomes following damage to prefrontal cortex. Cerebral Cortex, 6, 215-225.

ButTer, C. M. (1964). Habituation of responses to novel stimuli in monkeys with selective frontal lesions. Science, 144, 313-315.

Butter, C. M., McDonald, J. A., \& Snyder, D. R. (1969). Orality, preference behavior, and reinforcement value of nonfood objects in monkeys with orbital frontal lesions. Science, 164, 1306-1307.

Chao, L. L., \& Knight, R. T. (1996). Prefrontal and posterior cortical activation during auditory working memory. Cognitive Brain Research, 4, 27-37.

DAmasio, A. R. (1998). The somatic marker hypothesis and the possible functions of the prefrontal cortex. In A. C. Roberts, T. W. Robbins, \& L. Weiskrantz (Eds.), The prefrontal cortex: Executive and cognitive function (pp. 103-116). Oxford: Oxford University Press.

Damasio, A. R. Tranel, D., \& Damasio, H. (1990). Individuals with sociopathic behavior caused by frontal damage fail to respond autonomically to social stimuli. Behavioural Brain Research, 41, 81-94.

Davidson, R. J., Putnam, K. M., \& Larson, C. L. (2000). Dysfunction in the neural circuitry of emotion regulation: A possible prelude to violence. Science, 289, 591-594.

D’Esposito, M., Post le, B. R., Ballard, D., \& Lease, J. (1999). Maintenance versus manipulation of information held in working memory: An event-related fMRI study. Brain \& Cognition, 41, 66-86.

Dias, R, Robbins, T. W., \& Roberts, A. C. (1996). Dissociation in prefrontal cortex of affective and attentional shifts. Nature, 380, 69-72.

Harlow, J. M. (1848). Passage of an iron rod through the head. Boston Medical \& Surgical Journal, 39, 389-393.

Harlow, J. M. (1868). Recovery of an iron rod through the head. Publications of the Massachusetts Medical Society, 2, 327-347.

Hartikainen, K., Ogawa, K. H., Soltani, M., Pepitone, M., \& KNIGHT, R. T. (2000). Altered emotional influence on visual attention subsequent to orbitofrontal damage in humans. Society for Neuroscience Abstracts, 26, 2023.

IvERSEN, S. D., \& MishKin, M. (1970). Perseverative interference in monkeys following selective lesions of the inferior prefrontal convexity. Experimental Brain Research, 11, 376-386.

Kaipio, M.-L., Alho, K., Winkler, I., Escera, C., Surma-Aho, O., \& NÄÄTÄNEN, R. (1999). Event-related brain potentials reveal covert distractibility in closed head injuries. NeuroReport, 10, 2125-2159.

KNIGHT, R. T. (1984). Decreased response to novel stimuli after prefrontal lesions in man. Electroencephalography \& Clinical Neurophysiology, 59, 9-20.

KNIGHT, R. T. (1996). Contribution of human hippocampal region to novelty detection. Nature, 383, 256-259.

Knight, R. T., Scabini, D., Woods, D. L., \& Clayworth, C. C. (1989). Contributions of temporal-parietal junction to the human auditory P3. Brain Research, 502, 109-616.

Knight, R. T., Staines, W. R., Swick, D. , \& Chao, L. L. (1999). Prefrontal cortex regulates inhibition and excitation in distributed neural networks. Acta Psychologica, 101, 159-178.

LeDoux, J. E. (2000). Emotion circuits in the brain. Annual Review of Neuroscience, 23, 155-184.

Macmillan, M. (1986). A wonderful journey through skull and brains: The travels of Mr. Gage's tamping iron. Brain \& Cognition, 5, 67-107.
Macmillan, M. (1999). An odd kind of fame: Stories of Phineas Gage. Cambridge, MA: MIT Press, Bradford Books.

Mattson, A. J., \& Levin, H. S. (1990). Frontal lobe dysfunction following closed head injury. Journal of Nervous \& Mental Disease, 178, 282-291.

McGaUgh, J. L. (2000). Memory: A century of consolidation. Science, 287, 248-251.

Miller, E. K., \& Cohen, J. D. (2001). An integrative theory of prefrontal cortex function. Annual Review of Neuroscience, 24, 167-202.

Opitz, B., Mecklinger, A., Friederici, A. D., \& von Cramon, D. Y. (1999). The functional neuroanatomy of novelty processing: Integrating ERP and fMRI results. Cerebral Cortex, 9, 379-391.

PANKSEPP, J. (1998). Affective neuroscience: The foundations of human and animal emotions. New York: Oxford University Press.

Petrides, M. (1998). Specialized systems for the processing of mnemonic information within the primate frontal cortex. In A. C. Roberts, T. W. Robbins, \& L. Weiskrantz (Eds.), The prefrontal cortex: Executive and cognitive function (pp. 103-116). Oxford: Oxford University Press.

Petrides, M., \& Pandya, D. N. (1994). Comparative architectonic analysis of the human and the macaque frontal cortex. In F. Boller \& J. Grafman (Eds.), Handbook of neuropsychology (Vol. 9, pp. 17-58). Amsterdam: Elsevier.

Puce, A., Constable, R. T., Luby, M. L., McCarthy, G., Nobre, A. C., Spencer, D. D., Gore, J. C., \& Allison, T. (1995). Functional magnetic resonance imaging of sensory and motor cortex: Comparison with electrophysiological localization. Journal of Neurosurgery, 83, 262-270.

Roberts, N. A., Levens, S. M., McCoy, K., Werner, K., Beer, J. S., SCABINI, D., \& KNIGHT, R. T. (2001). Orbitofrontal cortex and activation of defensive responses. Society for Neuroscience Abstracts, 27, 1705.

Rolls, E. T. (2000). The orbitofrontal cortex and reward. Cerebral Cortex, 10, 284-294.

Rolls, E. T., Hornak, J., Wade, D., \& McGrath, J. (1994). Emotionrelated learning in patients with social and emotional changes associated with frontal lobe damage. Journal of Neurology, Neurosurgery \& Psychiatry, 57, 1518-1524.

Shallice, T., \& Burgess, P. (1993). Supervisory control of action and thought selection. In A. Baddeley \& L. Weiskrantz (Eds.), Attention: Selection, awareness, and control: A tribute to Donald Broadbent (pp. 171-187). New York: Oxford University Press, Clarendon Press.

Shimamura, A. P. (2000). The role of the prefrontal cortex in dynamic filtering. Psychobiology, 28, 207-218.

Shimamura, A. P. (in press). Muybridge in motion: Travels in art, psychology, and neurology. History of Photography.

SMITH, E. E., \& JonIDES, J. (1999). Storage and executive processes in the frontal lobes. Science, 283, 1657-1661.

Solbakk, A. K., Reinvang, I., Nielsen, C., \& Sundet, K. (1999). ERP indicators of disturbed attention in mild closed head injury: A frontal lobe syndrome? Psychophysiology, 36, 802-817.

Soltani, M., \& KNIGHT, R.T. (in press). Neural origins of the P300. Critical Reviews in Neurobiology.

(Manuscript received February 11, 2002; revision accepted for publication August 13, 2002.) 